\title{
ACTIVE CONTROL DESIGN FOR THE HYBRID SYNCHRONIZATION OF ARNEODO AND RÖSSLER CHAOTIC SYSTEMS
}

\author{
Sundarapandian Vaidyanathan ${ }^{1}$ and Suresh Rasappan ${ }^{2}$ \\ ${ }^{1}$ Research and Development Centre, Vel Tech Dr. RR \& Dr. SR Technical University \\ Avadi, Chennai-600 062, Tamil Nadu, INDIA \\ sundarvtu@gmail. com \\ ${ }^{2}$ Department of Mathematics, Vel Tech Dr. RR \& Dr. SR Technical University \\ Avadi, Chennai-600 062, Tamil Nadu, INDIA \\ mrpsuresh83@gmail.com
}

\begin{abstract}
This paper investigates the hybrid chaos synchronization of identical Arneodo systems (1981), identical Rössler systems (1976) and non-identical Arneodo and Rössler systems. In hybrid synchronization of chaotic systems, one part of the systems is synchronized, while the other part is anti-synchronized so that complete synchronization $(C S)$ and anti-synchronization $(A S)$ co-exist in the systems. The co-existence of $C S$ and $A S$ is very useful in secure communication and chaotic encryption schemes. Active nonlinear control method is used for hybrid synchronization of the Arneodo and Rössler systems and the synchronization results have been proved using Lyapunov stability theory. Since the Lyapunov exponents are not required for these calculations, the active control method is very effective and convenient to achieve hybrid synchronization of the Arneodo and Rössler systems. Numerical simulations are shown to verify the results.
\end{abstract}

\section{KEYWORDS}

Active Control, Hybrid Synchronization, Chaos, Chaotic Systems, Arneodo System, Rössler System.

\section{INTRODUCTION}

Chaotic systems are dynamical systems that are highly sensitive to initial conditions. The sensitive nature of chaotic systems is commonly called as the butterfly effect [1]. Chaos is an interesting nonlinear phenomenon and has been extensively and intensively studied in the last two decades [1-23]. Chaos theory has been applied in many scientific disciplines such as Mathematics, Computer Science, Microbiology, Biology, Ecology, Economics, Population Dynamics and Robotics.

In 1990, Pecora and Carroll [2] introduced a method to synchronize two identical chaotic systems and showed that it was possible for some chaotic systems to be completely synchronized. From then on, chaos synchronization has been widely explored in a variety of fields including physical [3], chemical [4], ecological [5] systems, secure communications [6-7], etc.

DOI : $10.5121 /$ cseij.2012.2202 
In most of the chaos synchronization approaches, the master-slave or drive-response formalism has been used. If a particular chaotic system is called the master or drive system and another chaotic system is called the slave or response system, then the idea of synchronization is to use the output of the master system to control the slave system so that the output of the slave system tracks the output of the master system asymptotically.

Since the seminal work by Pecora and Carroll [2], a variety of impressive approaches have been proposed for the synchronization of chaotic systems such as the sampled-data feedback synchronization method [8], OGY method [9], time-delay feedback method [10], backstepping method [11], adaptive design method [12], sliding mode control method [13], etc.

So far, many types of synchronization phenomenon have been presented such as complete synchronization [2], phase synchronization [5, 14], generalized synchronization [7, 15], antisynchronization [16, 17], projective synchronization [18], generalized projective synchronization $[19,20]$, etc.

Complete synchronization (CS) is characterized by the equality of state variables evolving in time, while anti-synchronization (AS) is characterized by the disappearance of the sum of relevant variables evolving in time. Projective synchronization (PS) is characterized by the fact that the master and slave systems could be synchronized up to a scaling factor, whereas in generalized projective synchronization (GPS), the responses of the synchronized dynamical states synchronize up to a constant scaling matrix $\alpha$. It is easy to see that the complete synchronization (CS) and anti-synchronization (AS) are special cases of the generalized projective synchronization (GPS) where the scaling matrix $\alpha=I$ and $\alpha=-I$, respectively.

In hybrid synchronization of chaotic systems [20], one part of the system is synchronized and the other part is anti-synchronized so that the complete synchronization (CS) and antisynchronization (AS) coexist in the system. The coexistence of CS and AS is highly useful in secure communication and chaotic encryptation schemes.

This paper is organized as follows. In Section 2, we derive results for the hybrid synchronization of identical Arneodo systems ([22], 1981). In Section 3, we derive results for the hybrid synchronization of identical Rössler systems ([23], 1976). In Section 4, we derive results for the hybrid synchronization of non-identical Arneodo and Rössler systems. The nonlinear controllers are derived using Lyapunov stability theory for the hybrid synchronization of the two chaotic systems. The proposed active control method is simple, effective and easy to implement in practical applications. Conclusions are contained in the final section.

\section{HYBRID SYNCHRONIZATION OF IDENTICAL QI SYSTEMS}

In this section, we consider the hybrid synchronization of identical Arneodo chaotic systems ([22], 1981).

Thus, we consider the master system as the Arneodo dynamics described by

$$
\begin{aligned}
& \dot{x}_{1}=x_{2} \\
& \dot{x}_{2}=x_{3} \\
& \dot{x}_{3}=m x_{1}-s x_{2}-x_{3}-x_{1}^{2}
\end{aligned}
$$

where $x_{i},(i=1,2,3)$ are the state variables and $s, m$ are positive constants.

The Arneodo system (1) is chaotic when $s=3.8$ and $m=7.5$. 
The chaotic attractor of the Arneodo system is shown in Figure 1.

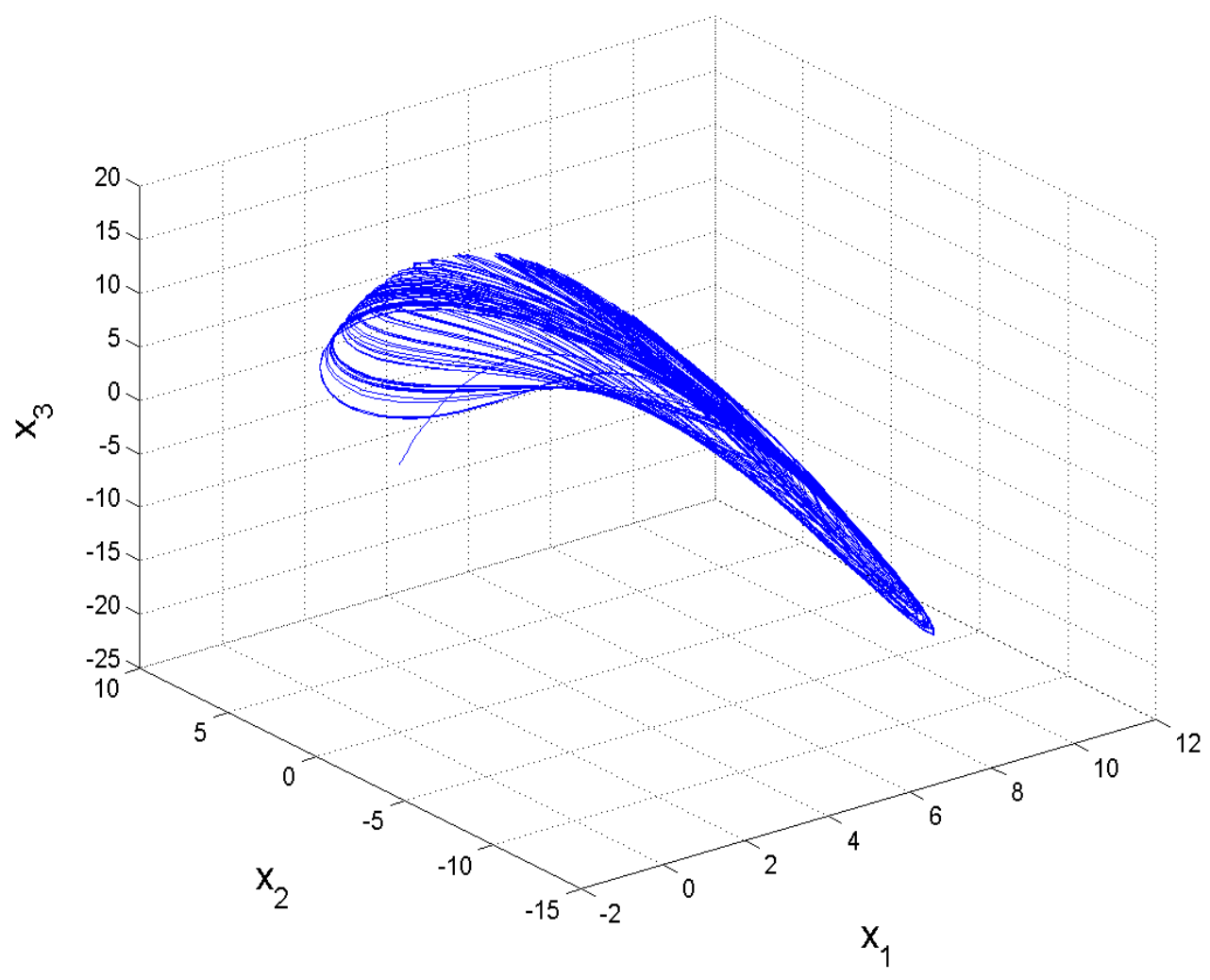

Figure 1. The Arneodo Chaotic Attractor

We consider the Arneodo dynamics also as the slave system, which is described by

$$
\begin{aligned}
& \dot{y}_{1}=y_{2}+u_{1} \\
& \dot{y}_{2}=y_{3}+u_{2} \\
& \dot{y}_{3}=m y_{1}-s y_{2}-y_{3}-y_{1}^{2}+u_{3}
\end{aligned}
$$

where $y_{i},(i=1,2,3)$ are the state variables and $u_{i},(i=1,2,3)$ are the active controls.

For the hybrid synchronization of the identical Arneodo systems (1) and (2), the synchronization errors are defined as

$$
\begin{aligned}
& e_{1}=y_{1}-x_{1} \\
& e_{2}=y_{2}+x_{2} \\
& e_{3}=y_{3}-x_{3}
\end{aligned}
$$

A simple calculation yields the error dynamics as 


$$
\begin{aligned}
& \dot{e}_{1}=e_{2}-2 x_{2}+u_{1} \\
& \dot{e}_{2}=e_{3}+2 x_{3}+u_{2} \\
& \dot{e}_{3}=m e_{1}-s e_{2}-e_{3}+2 s x_{2}-y_{1}^{2}+x_{1}^{2}+u_{3}
\end{aligned}
$$

We consider the active nonlinear controller defined by

$$
\begin{aligned}
& u_{1}=-e_{2}+2 x_{2}-k_{1} e_{1} \\
& u_{2}=-e_{3}-2 x_{3}-k_{2} e_{2} \\
& u_{3}=-m e_{1}+s e_{2}-2 s x_{2}+y_{1}^{2}-x_{1}^{2}
\end{aligned}
$$

where $k_{1}$ and $k_{2}$ are positive constants.

Substitution of (5) into (4) yields the linear error dynamics

$$
\begin{aligned}
& \dot{e}_{1}=-k_{1} e_{1} \\
& \dot{e}_{2}=-k_{2} e_{2} \\
& \dot{e}_{3}=-e_{3}
\end{aligned}
$$

We consider the candidate Lyapunov function defined by

$$
V(e)=\frac{1}{2} e^{T} e=\frac{1}{2}\left(e_{1}^{2}+e_{2}^{2}+e_{3}^{2}\right)
$$

which is a positive definite function on $R^{3}$.

Differentiating (7) along the trajectories of the system (6), we get

$$
\dot{V}(e)=-k_{1} e_{1}^{2}-k_{2} e_{2}^{2}-e_{3}^{2}
$$

which is a negative definite function on $R^{3}$ since $k_{1}$ and $k_{2}$ are positive constants.

Thus, by Lyapunov stability theory [24], the error dynamics (6) is globally exponentially stable. Hence, we obtain the following result.

Theorem 1. The identical Arneodo systems (1) and (2) are globally and exponentially hybrid synchronized with the active nonlinear controller (5).

\section{Numerical Simulations}

For the numerical simulations, the fourth order Runge-Kutta method is used to solve the two systems of differential equations (1) and (2) with the nonlinear controller (5).

The parameters of the identical Arneodo systems (1) and (2) are selected as $s=3.8$ and $m=7.5$ so that the systems exhibit chaotic behaviour. Also, we take $k_{1}=3$ and $k_{2}=3$.

The initial values for the master system (1) are taken as 


$$
x_{1}(0)=10, x_{2}(0)=-5, \quad x_{3}(0)=12
$$

The initial values for the slave system (2) are taken as

$$
y_{1}(0)=5, \quad y_{2}(0)=3, \quad y_{3}(0)=-4
$$
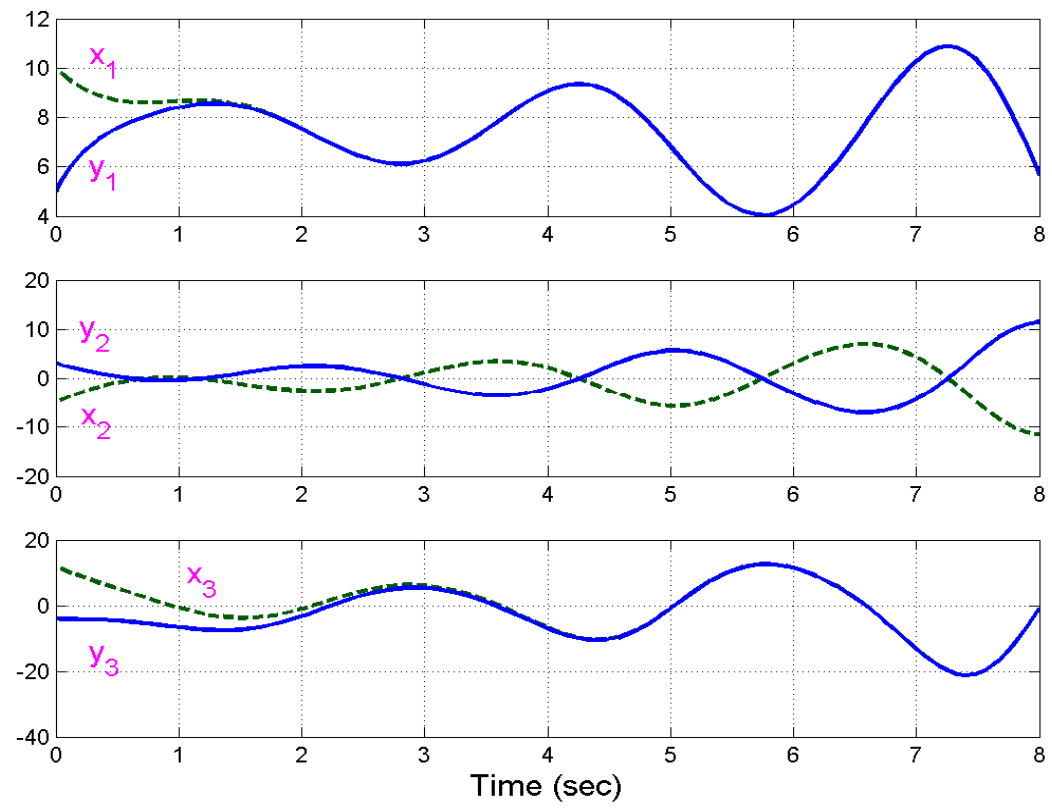

Figure 2 exhibits the hybrid synchronization of the chaotic systems (1) and (2).

Figure 2. Hybrid Synchronization of the Identical Arneodo Systems

\section{HYBRID SYNCHRONIZATION OF IDENTICAL RÖSSLER SYSTEMS}

In this section, we consider the hybrid synchronization of identical Rössler systems ([23], 1976).

Thus, we consider the master system as the Rössler dynamics described by

$$
\begin{aligned}
& \dot{x}_{1}=-x_{2}-x_{3} \\
& \dot{x}_{2}=x_{1}+a x_{2} \\
& \dot{x}_{3}=b+\left(x_{1}-c\right) x_{3}
\end{aligned}
$$

where $x_{i},(i=1,2,3)$ are the state variables and $a, b, c$ are positive constants.

The system (8) is chaotic, when the parameter values are taken as $a=0.2, b=0.2$ and $c=5.7$. 


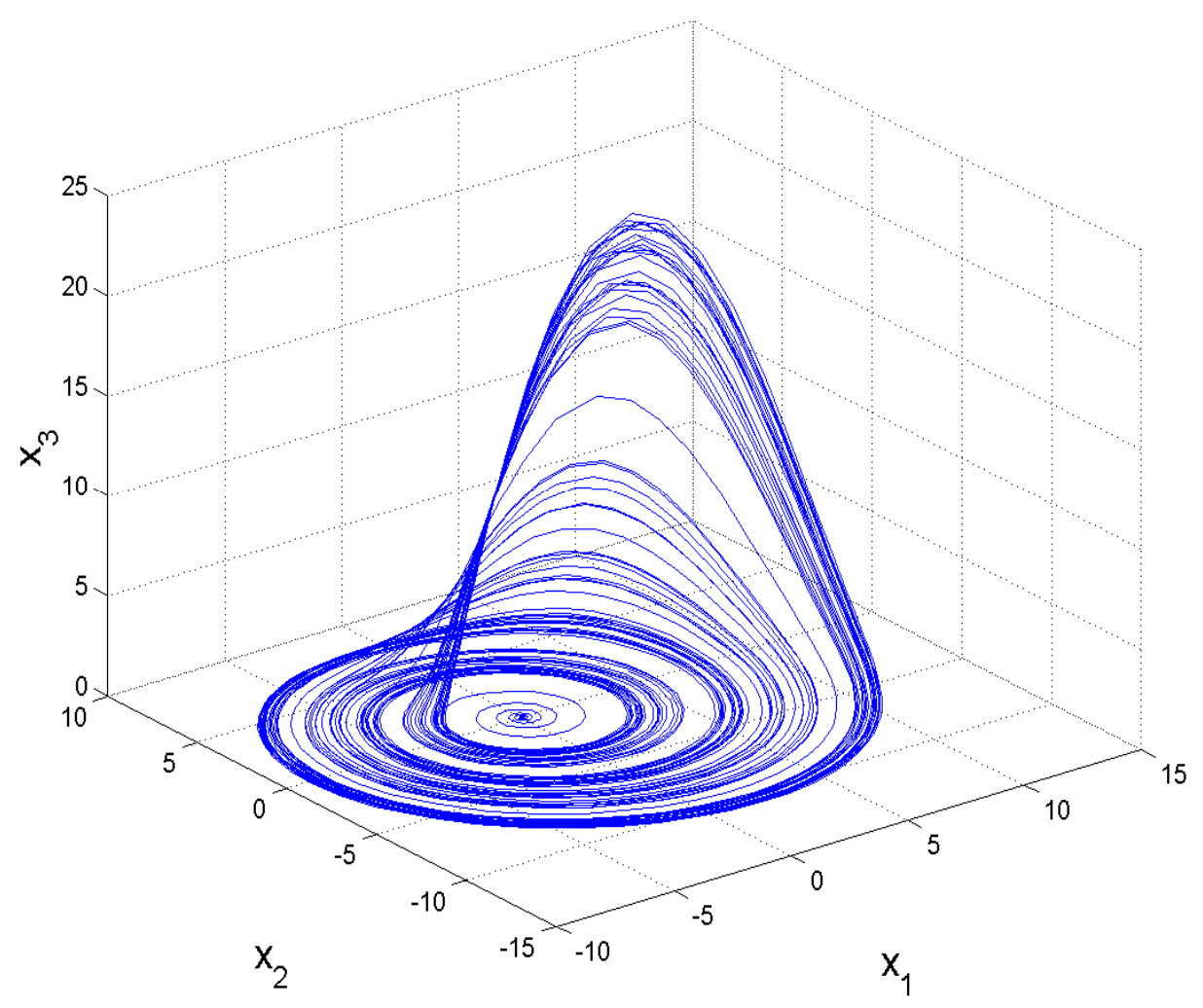

Figure 3. The Rössler Chaotic Attractor

Figure 3 depicts the strange attractor of the Rössler system (8).

We consider the controlled Rössler dynamics also as the slave system, which is described by

$$
\begin{aligned}
& \dot{y}_{1}=-y_{2}-y_{3}+u_{1} \\
& \dot{y}_{2}=y_{1}+a y_{2}+u_{2} \\
& \dot{y}_{3}=b+\left(y_{1}-c\right) y_{3}+u_{3}
\end{aligned}
$$

where $y_{i},(i=1,2,3)$ are the state variables and $u_{i},(i=1,2,3)$ are the active controls.

For the hybrid synchronization of the identical Rössler systems (8) and (9), the synchronization errors are defined as

$$
\begin{aligned}
& e_{1}=y_{1}-x_{1} \\
& e_{2}=y_{2}+x_{2} \\
& e_{3}=y_{3}-x_{3}
\end{aligned}
$$

A simple calculation yields the error dynamics as 


$$
\begin{aligned}
& \dot{e}_{1}=-e_{2}-e_{3}+2 x_{2}+u_{1} \\
& \dot{e}_{2}=e_{1}+a e_{2}+2 x_{1}+u_{2} \\
& \dot{e}_{3}=-c e_{3}+y_{1} y_{3}-x_{1} x_{3}+u_{3}
\end{aligned}
$$

We consider the active nonlinear controller defined by

$$
\begin{aligned}
& u_{1}=e_{2}+e_{3}-2 x_{2}-k_{1} e_{1} \\
& u_{2}=-e_{1}-a e_{2}-2 x_{1}-k_{2} e_{2} \\
& u_{3}=-y_{1} y_{3}+x_{1} x_{3}
\end{aligned}
$$

where $k_{1}$ and $k_{2}$ are positive constants.

Substitution of (12) into (11) yields the linear error dynamics

$$
\begin{aligned}
& \dot{e}_{1}=-k_{1} e_{1} \\
& \dot{e}_{2}=-k_{2} e_{2} \\
& \dot{e}_{3}=-c e_{3}
\end{aligned}
$$

We consider the candidate Lyapunov function defined by

$$
V(e)=\frac{1}{2} e^{T} e=\frac{1}{2}\left(e_{1}^{2}+e_{2}^{2}+e_{3}^{2}\right)
$$

which is a positive definite function on $R^{3}$.

Differentiating (14) along the trajectories of the system (13), we get

$$
\dot{V}(e)=-k_{1} e_{1}^{2}-k_{2} e_{2}^{2}-c e_{3}^{2},
$$

which is a negative definite function on $R^{3}$.

Thus, by Lyapunov stability theory [24], the error dynamics (13) is globally exponentially stable. Hence, we obtain the following result.

Theorem 2. The identical Rössler systems (8) and (9) are globally and exponentially hybrid synchronized with the active nonlinear controller (12).

\section{Numerical Simulations}

For the numerical simulations, the fourth order Runge-Kutta method is used to solve the two systems of differential equations (8) and (9) with the nonlinear controller (12).

The parameters of the identical Rössler systems (8) and (9) are selected as

$$
a=0.2, b=0.2, c=5.7
$$


so that the systems (8) and (9) exhibit chaotic behaviour.

We take the gains as

$$
k_{1}=3 \text { and } k_{2}=3 \text {. }
$$

The initial values for the master system (8) are taken as

$$
x_{1}(0)=6, \quad x_{2}(0)=15, \quad x_{3}(0)=12
$$

The initial values for the slave system (9) are taken as

$$
y_{1}(0)=1, \quad y_{2}(0)=10, y_{3}(0)=3
$$

Figure 4 exhibits the hybrid synchronization of the Rössler chaotic systems (8) and (9).
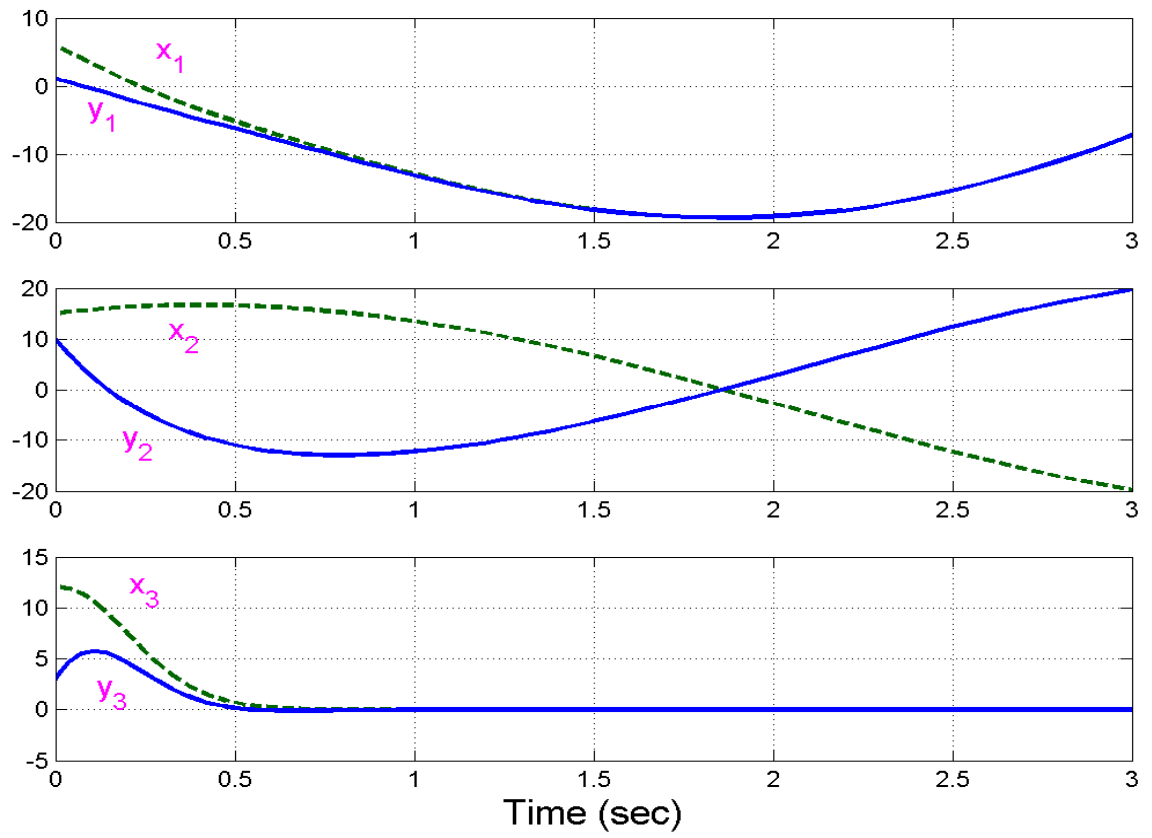

Figure 4. Hybrid Synchronization of the Identical Rössler Systems

\section{Hybrid SyNCHRONIZATION OF ARNEODO AND RÖSSLER SYSTEMS}

In this section, we consider the hybrid synchronization of non-identical Arneodo and Rössler systems. We take the Arneodo system as the master system and the Rössler system as the slave system in the hybrid synchronization of non-identical chaotic systems.

Thus, we consider the master system as the Arneodo dynamics described by 


$$
\begin{aligned}
& \dot{x}_{1}=x_{2} \\
& \dot{x}_{2}=x_{3} \\
& \dot{x}_{3}=m x_{1}-s x_{2}-x_{3}-x_{1}^{2}
\end{aligned}
$$

where $x_{i},(i=1,2,3)$ are the state variables and $s, m$ are positive constants.

We consider the Rössler system as the slave system, which is described by

$$
\begin{aligned}
& \dot{y}_{1}=-y_{2}-y_{3}+u_{1} \\
& \dot{y}_{2}=y_{1}+a y_{2}+u_{2} \\
& \dot{y}_{3}=b+\left(y_{1}-c\right) y_{3}+u_{3}
\end{aligned}
$$

where $y_{i},(i=1,2,3)$ are the state variables, $a, b, c$ are positive constants and $u_{i},(i=1,2,3)$ are the active controls.

For the hybrid synchronization of the chaotic systems (15) and (16), the synchronization errors are defined as

$$
\begin{aligned}
& e_{1}=y_{1}-x_{1} \\
& e_{2}=y_{2}+x_{2} \\
& e_{3}=y_{3}-x_{3}
\end{aligned}
$$

A simple calculation yields the error dynamics as

$$
\begin{aligned}
& \dot{e}_{1}=-e_{2}-y_{3}+u_{1} \\
& \dot{e}_{2}=e_{1}+x_{1}+x_{3}+a y_{2}+u_{2} \\
& \dot{e}_{3}=b-c e_{3}-m x_{1}+s x_{2}+(1-c) x_{3}+x_{1}^{2}+y_{1} y_{3}+u_{3}
\end{aligned}
$$

We consider the nonlinear controller defined by

$$
\begin{aligned}
& u_{1}=e_{2}+y_{3}-k_{1} e_{1} \\
& u_{2}=-e_{1}-x_{1}-x_{3}-a y_{2}-k_{2} e_{2} \\
& u_{3}=-b+m x_{1}-s x_{2}-(1-c) x_{3}-x_{1}^{2}-y_{1} y_{3}
\end{aligned}
$$

where $k_{1}$ and $k_{2}$ are positive constants.

Substitution of (19) into (18) yields the linear error dynamics

$$
\begin{aligned}
& \dot{e}_{1}=-k_{1} e_{1} \\
& \dot{e}_{2}=-k_{2} e_{2} \\
& \dot{e}_{3}=-c e_{3}
\end{aligned}
$$

We consider the candidate Lyapunov function defined by 


$$
V(e)=\frac{1}{2} e^{T} e=\frac{1}{2}\left(e_{1}^{2}+e_{2}^{2}+e_{3}^{2}\right)
$$

which is a positive definite function on $R^{3}$.

Differentiating (21) along the trajectories of the system (20), we get

$$
\dot{V}(e)=-k_{1} e_{1}^{2}-k_{2} e_{2}^{2}-c e_{3}^{2},
$$

which is a negative definite function on $R^{3}$.

Thus, by Lyapunov stability theory [24], the error dynamics (20) is globally exponentially stable. Hence, we obtain the following result.

Theorem 3. The non-identical Arneodo system (15) and Rössler system (16) are globally and exponentially hybrid synchronized with the active nonlinear controller (19).

\section{Numerical Simulations}

For the numerical simulations, the fourth order Runge-Kutta method is used to solve the two systems of differential equations (15) and (16) with the nonlinear controller (19).

The parameters of the Arneodo system (15) are chosen as $s=3.8$ and $m=7.5$.

The parameters of the Rössler system (16) are chosen as $a=0.2, b=0.2$ and $c=5.7$.

We take the gains as $k_{1}=3$ and $k_{2}=3$.

The initial values for the master system (15) are taken as

$$
x_{1}(0)=2, \quad x_{2}(0)=10, \quad x_{3}(0)=5
$$

The initial values for the slave system (16) are taken as

$$
y_{1}(0)=16, \quad y_{2}(0)=3, \quad y_{3}(0)=11
$$

Figure 5 exhibits the hybrid synchronization of the chaotic systems (15) and (16). 

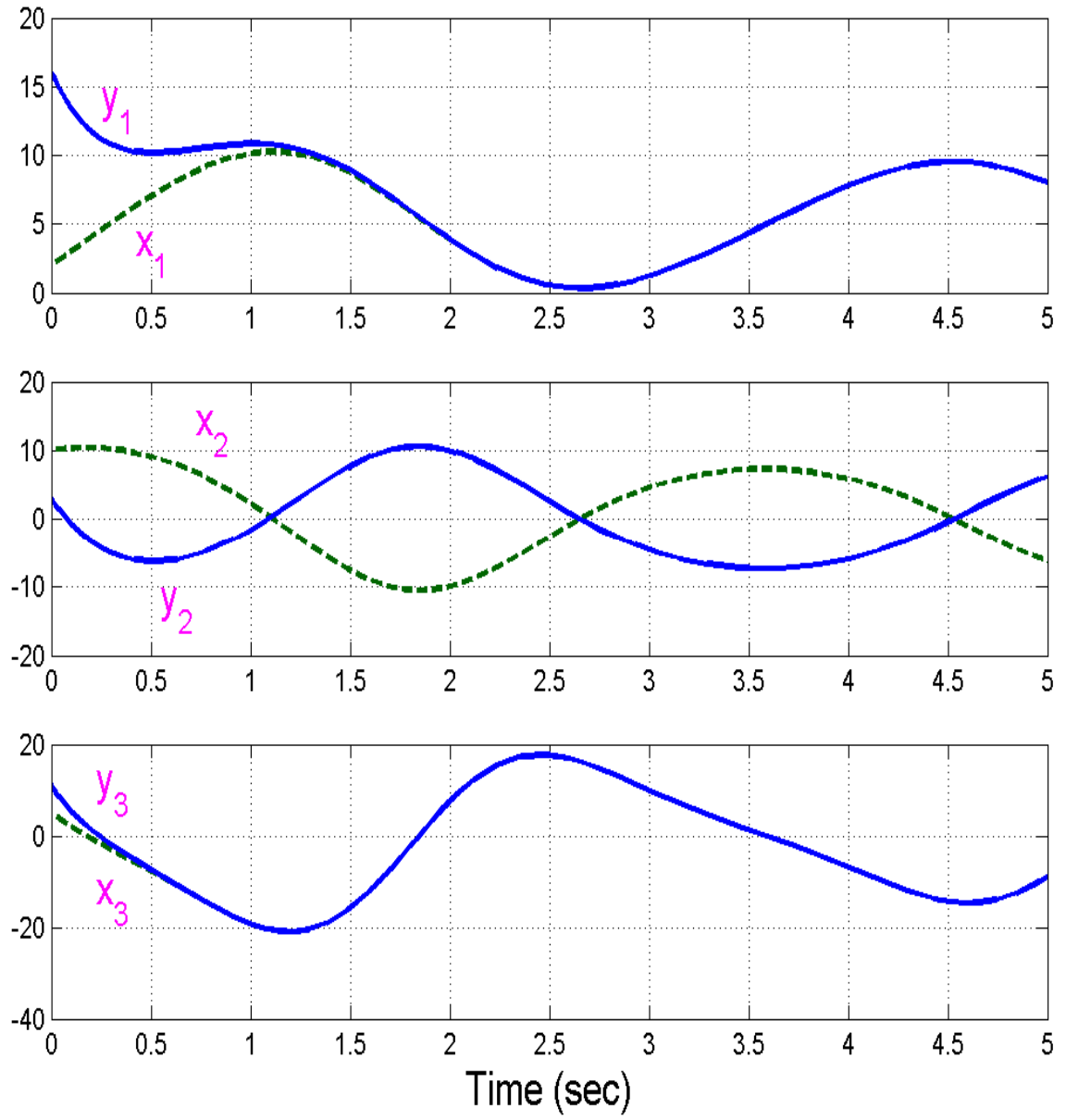

Figure 5. Hybrid Synchronization of Arneodo and Rössler Systems

\section{Conclusions}

In this paper, active control method has been deployed to achieve hybrid chaos synchronization of the following chaotic systems:

(A) Two identical Arneodo systems (1981)

(B) Two identical Rössler systems (1976)

(C) Non-identical Arneodo and Rössler systems.

The synchronization results were derived using active control method and were established using Lyapunov stability theory. Since Lyapunov exponents are not required for these calculations, the proposed active control method is effective and convenient to achieve hybrid synchronization of the chaotic systems as mentioned in the three cases, (A)-(C). Numerical simulations have been given to demonstrate the effectiveness of the proposed hybrid synchronization schemes. 
Computer Science \& Engineering: An International Journal (CSEIJ), Vol.2, No.2, April 2012

\section{REFERENCES}

[1] Alligood, K.T., Sauer, T. \& Yorke, J.A. (1997) Chaos: An Introduction to Dynamical Systems, Springer, New York, U.S.A.

[2] Pecora, L.M. \& Carroll, T.L. (1990) "Synchronization in chaotic systems", Physical Review Letters, Vol. 64, pp 821-824.

[3] Lakshmanan, M. \& Murali, K. (1996) Nonlinear Oscillators: Controlling and Synchronization, World Scientific, Singapore.

[4] Han, S.K., Kerrer, C. \& Kuramoto, Y. (1995) "Dephasing and burstling in coupled neural oscillators", Phys. Rev. Lett., Vol. 75, pp 3190-3193.

[5] Blasius, B., Huppert, A. \& Stone, L. (1999) "Complex dynamics and phase synchronization in spatially extended ecological system”, Nature, Vol. 399, pp 354-359.

[6] Feki, M. (2003) "An adaptive chaos synchronization scheme applied to secure communication", Chaos, Solitons and Fractals, Vol. 18, pp 141-148.

[7] Murali, K. \& Lakshmanan, M. (1998) "Secure communication using a compound signal from generalized synchronizable chaotic systems", Phys. Rev. Lett. A, Vol. 241, pp 303-310.

[8] Yang, T. \& Chua, L.O. (1999) "Control of chaos using sampled-data feedback control", Internat. J. Bifurcat. Chaos, Vol. 9, pp 215-219.

[9] Ott, E., Grebogi, C. \& Yorke, J.A. (1990) “Controlling chaos”, Phys. Rev. Lett., Vol. 64, pp 11961199.

[10] Park, J.H. \& Kwon, O.M. (2003) "A novel criterion for delayed feedback control of time-delay chaotic systems", Chaos, Solitons and Fractals, Vol. 17, pp 709-716.

[11] Yu, Y.G. \& Zhang, S.C. (2006) "Adaptive backstepping synchronization of uncertain chaotic systems", Chaos, Solitons and Fractals, Vol. 27, pp 1369-1375.

[12] Liao, T.L. \& Tsai, S.H. (2000) "Adaptive synchronization of chaotic systems and its applications to secure communications", Chaos, Solitons and Fractals, Vol. 11, pp 1387-1396.

[13] Konishi, K.., Hirai, M. \& Kokame, H. (1998) "Sliding mode control for a class of chaotic systems", Phys. Lett. A, Vol. 245, pp 511-517.

[14] Ge, Z.M. \& Chen, C.C. (2004) "Phase synchronization of coupled chaotic multiple time scales systems", Chaos, Solitons and Fractals, Vol. 20, pp 639-647.

[15] Wang, Y.W. \& Guan, Z.H. (2006) "Generalized synchronization of continuous chaotic systems", Chaos, Solitons and Fractals, Vol. 27, pp 97-101.

[16] Zhang, X. \& Zhu, H. (2008) "Anti-synchronization of two different hyperchaotic systems via active and adaptive control”, Internat. J. Nonlinear Science, Vol. 6, pp 216-223.

[17] Chiang, T., Lin, J., Liao, T. \& Yan, J. (2008) "Anti-synchronization of uncertain unified chaotic systems with dead-zone nonlinearity”, Nonlinear Analysis, Vol. 68, pp 2629-2637.

[18] Qiang, J. (2007) "Projective synchronization of a new hyperchaotic Lorenz system", Phys. Lett. A, Vol. 370, pp 40-45.

[19] Jian-Ping, Y. \& Chang-Pin, L. (2006) "Generalized projective synchronization for the chaotic Lorenz system and the chaotic Chen system", J. Shanghai Univ., Vol. 10, pp 299-304.

[20] Li, R.H., Xu, W. \& Li, S. (2007) "Adaptive generalized projective synchronization in different chaotic systems based on parameter identification”, Phys. Lett. A, Vol. 367, pp 199-206.

[21] Li, R-H. (2008) “A special full-state hybrid synchronization in symmetrical chaotic systems", Applied Math. Comput., Vol. 200, pp 321-319.

[22] Arneodo, A., Coullet, P. \& Tresser, C. (1981) "Possible new strange attractors with spiral structure," Commun. Math. Physics, Vol. 79, pp 573-579.

[23] Rössler, O.E. (1976) “An equation for continuous chaos,” Physics Letters A, Vol. 57, pp 397-398.

[24] Hahn, W. (1967) The Stability of Motion, Springer, New York, U.S.A. 
Computer Science \& Engineering: An International Journal (CSEIJ), Vol.2, No.2, April 2012

\section{Authors}

Dr. V. Sundarapandian obtained his Doctor of Science degree in Electrical and Systems Engineering from Washington University, Saint Louis, USA under the guidance of Late Dr. Christopher I. Byrnes (Dean, School of Engineering and Applied Science) in 1996. He is currently Professor in the Research and Development Centre at Vel Tech Dr. RR \& Dr. SR Technical University, Chennai, Tamil Nadu, India. He has published over 240 refereed international publications. He has published over 100 papers in National Conferences and over 60 papers in International Conferences. He is the Editor-in-Chief of International Journal of Mathematics and Scientific Computing, International Journal of Instrumentation and Control Systems, International Journal of Control Systems and Computer Modelling, etc. His research interests are Linear and Nonlinear Control Systems, Chaos Theory and Control, Soft Computing, Optimal Control, Process Control, Operations Research, Mathematical Modelling and Scientific Computing using MATLAB. He has delivered many Key Note Lectures on Control Systems, Chaos, Scientific Computing, Modelling and Simulation with SCILAB, MATLAB, etc.

Mr. R. Suresh obtained his M.Sc degree in Mathematics from Bharathiar University, Coimbatore, Tamil Nadu, India in 2005, and M.Phil degree in Mathematics from Bharathiar University, Coimbatore, Tamil Nadu, India in 2008. He is currently working as a Lecturer in the Department of Mathematics, Vel Tech Dr. RR \& Dr. SR Technical University, Chennai, India. He is pursuing his Ph.D. degree in Mathematics under the guidance of Research Professor, Dr. V. Sundarapandian in Vel Tech Dr. RR \& Dr. SR Technical University, Chennai, India. He has published over 10 papers in International

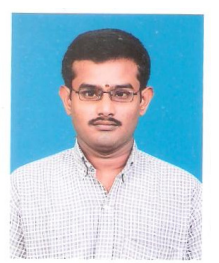
Journals. His research interests are in Chaos and Control. 